\title{
Efficient inhibition of ovarian cancer by short hairpin RNA targeting claudin-3
}

\author{
CHUNTANG SUN ${ }^{1}$, TAO YI $^{1}$, XIANGRONG SONG ${ }^{2}$, SHUANGZHI LI ${ }^{2}$, XIAORONG QI ${ }^{1}$, \\ XIANCHENG CHEN ${ }^{2}$, HONGGANG LIN ${ }^{2}$, XIANG HE $^{1}$, ZHENGYU LI $^{1}$, YUQUAN WEI $^{2}$ and XIAZHAO ${ }^{1}$ \\ ${ }^{1}$ Department of Gynecology and Obstetrics, West China Second Hospital, Sichuan University, Chengdu; \\ ${ }^{2}$ State Key Laboratory, Biotherapy and Cancer Center, West China Hospital, Sichuan University, Chengdu, P.R. China
}

Received February 10, 2011; Accepted March 29, 2011

DOI: $10.3892 / o r .2011 .1275$

\begin{abstract}
Ovarian cancer is one of the most lethal gynecologic neoplasms. Even though various new chemotherapeutics have been developed for the treatment of ovarian cancer, drug resistance and undesired serious side effects remain unavoidable obstacles for chemotherapeutic approaches. New strategies to overcome the therapeutic dilemma are needed. Claudin-3 (CLDN3) is a recently discovered gene generally overexpressed in human ovarian cancers but not in normal ovarian tissue. Its high expression has been identified to associate with the invasion, proliferation and survival of cancer cells, making it a promising target for gene therapy of ovarian cancer. However, in gene therapy, traditional gene carriers such as virus or cationic liposomes suffer from distressing shortcomings of potential carcinogenicity, obvious cytotoxicity and immunogenicity. Nanoparticles (NPs) based on PLGA are a novel gene delivery system with good biodegradability, excellent biocompatibility and low toxcity for in vivo gene delivery compared with traditional gene carriers. We constructed a plasmid expressing shRNA targeted CLDN3 (pshCLDN3) encapsulated with PLGA-NPs, and administered it by i.p. injection to nude mice bearing intraperitoneal SKOV3 ovarian cancer, to investigate the antitumor potential of knocking down CLDN3. After 12 times of administration, the tumors of each group were compared. The underlying antitumor mechanisms were revealed by immunostaining of CD31, Ki-67 and TUNEL assay, to exhibit possible alterations in microvessel density, cell proliferation and cell apoptosis. Our study demonstrated
\end{abstract}

Correspondence to: Professor Xia Zhao, Department of Gynecology and Obstetrics, West China Second Hospital of Sichuan University, No. 20, Section 3, South People's Road, Chengdu 610041, Sichuan, P.R. China

E-mail: xia-zhao@126.com

Abbreviations: CLDN3, claudin-3; PLGA, poly(lactic-co-glycolic acid); NPs, nanoparticles; NS, normal saline; shRNA, short hairpin RNA; RNAi, RNA interference

Key words: CLD N3, PLGA, nanoparticles, ovarian cancer, RNA interference, shRNA that i.p. administration of pshCLDN3 effectively suppressed the expression of CLDN3 and, thus, inhibited the growth of ovarian tumors, significantly reducing tumor weight by $67.4 \%$ compared with blank controls $(\mathrm{p}<0.05)$. Immunostaining of CD31, Ki-67 and TUNEL assay demonstrated decreased angiogenesis $(\mathrm{p}<0.05)$, reduced proliferation $(\mathrm{p}<0.05)$ and increased apoptosis $(\mathrm{p}<0.05)$ in the pshCLDN3 treated group compared with controls. No obvious toxicity of PLGA-NPs was observed either in vitro or in vivo. Our results indicated that knockdown of CLDN3 by pshCLDN3 encapsulated in PLGA NPs may provide a promising approach for the treatment of ovarian cancer.

\section{Introduction}

Ovarian cancer, which accounts for more than 50\% death of gynecologic malignancy, is the most lethal gynecologic carcinoma (1). Due to its few and imperceptible early symptoms, ovarian cancer is mostly at advanced stage in most patients during the initial diagnosis. Although patients may initially respond to the combination treatment of surgical and chemical therapies, most of them will inevitably die from relapse and the development of chemotherapy-resistant recurrence (2). The overall 5-year relative survival rate is no more than $50 \%$ (3). Therefore, new strategies to improve the treatment status of ovarian cancer are urgently needed.

Gene therapy is a novel therapeutic strategy different from traditional therapies. It exclusively targets the key gene for tumorigenesis, achieves therapeutic effect by regulating specific gene expression with few or negligible side effects. Besides the gene, gene carrier is another important factor that influences the safety and the ultimate effect of gene therapy (4). Traditional cationic liposomes and virus vectors show relatively better effect than other gene delivery materials in vitro, but many intrinsic shortcomings hinder their applications in vivo $(5,6)$. Therefore, it is essential to establish an efficient and safe gene delivery protocol. Nanoparticles (NPs) based on PLGA have been suggested as a promising carrier for DNA $(7,8)$. In addition to its biodegradability and biocompatibility, the potential of nanoparticles as a controlled release drug delivery system and protective agent of DNA against nucleinase has attracted much attention $(9,10)$. Previously, our lab has successfully 
prepared various PLGA NPs loaded with chemotherapeutic reagents $(11,12)$.

CLDN3 is an important member of claudin family involved in the formation of tight junction (TJ) of epithelial cells and cancer cells (13). It has been suggested as one of the most highly and consistently up-regulated genes in ovarian cancers $(14,15)$. The abnormal high expression of CLDN3 plays a crucial role in the proliferation, survival, invasion, cellular motility and metastasis of ovarian cancer, which makes CLDN3 a promising therapeutic target for the treatment of ovarian cancer (16). Recently, various new antitumor strategies involving CLDN3 have emerged, including therapeutic antibody, CPE meditated cytolysis and RNA interference (17-19). Among these, shRNA meditated knocking down of CLDN3 seemed of great prospect.

In this study, we adopted plasmid vector to express shRNA targeting CLDN3 (pshCLDN3), encapsulated it by PLGA-NPs (pshCLDN3-NPs), and then tested it both in vitro and in vivo, aimed to evaluate the antitumor effect of knocking down CLDN3 in ovarian cancer and explore the safety and potential of PLGA-NPs for gene administration in ovarian cancer treatment. In our study, pshCLDN3 induced specific and significant down-regulation of CLDN3 in human ovarian cancer SKOV3 cells in vitro. In vivo, administration of pshCLDN3-NPs to nude mice bearing human ovarian cancer significantly suppressed the growth of intraperitoneal metastatic tumor, with the possible mechanisms of antiangiogenesis, cell proliferation inhibition and apoptosis induction. These data showed that knocking down CLDN3 by shRNA meditated RNA interference might be a promising therapeutic approach for ovarian cancer.

\section{Materials and methods}

Vector construction and plasmid DNA extraction. According to shRNA design principles and published siRNA sequences for CLDN3, shRNA primers targeting CLDN3 (sense 5'-TCCCGCAACATCATCACGTCGCATTCAAGACGTG CGACGTGATGATGTTGCTTTTTTG-3' antisense, 5'-AGCTCAAAAAAGCAACATCATCACGTCGCACGT CTTGAATGCGACGTGATGATGTTGC-3') were designed and chemically synthesized for plasmid construction (19). The HK sequence, which has no homology with any known mammalian gene sequences, was used as the negative control. The pGenesil-2.1 plasmid vector (Genesil, Wuhan, China) with a kanamycin resistance gene was used as shRNA expression vector. Positive transformants containing pshCLDN3 or pshHK were isolated by kanamycin selection and validated by DNA sequencing. Large-scale preparation of plasmid DNA was purified using endotoxin-free Plasmid extract Giga kits (Qiagen, Germany).

Preparation and characterization of plasmid-loaded PLGA-NPs. The plasmid loaded PLGA-NPs (pDNAPLGA-NPs) were prepared by a modified method of w/o/w double-emulsion process of our lab $(20,21)$. The encapsulating efficiency of PLGA-NPs for pDNA was determined by the ratio of pDNA incorporated in PLGA-NPs to the total amount of pDNA in formulation (22). Particle size and zeta potential of pDNA-PLGA-NPs were determined using Zetasizer
Nano ZS90 (Malvern Instrument, UK). The cytotoxicity of PLGA-NPs was evaluated by methylthiazoletetrazolium (MTT) assay using HEK 293 cells. Viability of PLGA-NPs treated cells was assessed as percent cell viability in terms of media-only treated control cells. For cell uptake study, pDNA-PLGA-NPs were labeled with 6-coumarin (green) for subcellular localization. Labled pDNA-PLGA-NPs was incubated with SKOV3 cells and observed under inverted fluorescence microscope consecutively.

Cell culture and transfection. Human ovarian cancer SKOV3 cell line was purchased from American Type Culture Collection (ATCC, Manassas, VA, USA) and cultured in Dulbecco's modified Eagle's medium (DMEM) supplemented with $10 \%$ fetal bovine serum and antibiotics. Cells were incubated in a humidified atmosphere containing $5 \% \mathrm{CO}_{2}$ at $37^{\circ} \mathrm{C}$. For in vitro transfection, SKOV3 cells were seeded in 6 -well plates at a density of $1 \times 10^{5}$ cells/well and cultured to reach $40 \%$ confluence, then, $1 \mu \mathrm{g}$ of pshCLDN3 or pshHK encapsulated in PLGA-NPs was diluted using antibiotics-free DMEM medium to a final volume of $100 \mu \mathrm{l}$ and added to the corresponding wells. Equal volume of NS alone was used as the blank control. After incubating at $37^{\circ} \mathrm{C}$ for $72 \mathrm{~h}$, cells were harvested for further examination.

Western blot analysis. Western blot analysis was employed to determine the expression of CLDN3 in cells or tumor tissue. Cells or tumor tissue were lysed using RIPA lysis buffer [containing $50 \mathrm{mM}$ Tris- $\mathrm{HCl}(\mathrm{pH} 7.4), 0.25 \%$ sodium deoxycholate, $150 \mathrm{mM} \mathrm{NaCl}, 0.1 \%$ SDS, $1 \%$ NP-40, $1 \mathrm{mM}$ EDTA, $1 \mathrm{mM} \mathrm{NaF}, 1 \mathrm{mM} \mathrm{Na} \mathrm{VO}_{4}$ ], supplemented with proteinase inhibitor ( $1 \mathrm{mM}$ cocktail plus $1 \mathrm{mM}$ PMSF), then, lysed product was centrifuged at $15,000 \mathrm{rpm}, 4^{\circ} \mathrm{C}$, for $30 \mathrm{~min}$. Total Protein concentration in supernatant was determined using Bio-Rad protein assay kit (Bio-Rad Laboratories, Hercules, CA). Equal amount of protein $(40 \mu \mathrm{g})$ was loaded onto a $12 \%$ SDS-PAGE gel for electrophoresis and transferred onto polyvinylidene difluoride (PVDF) membrane (Millipore, Bedford, MA). After being blocked in 5\% skimmed milk for $1 \mathrm{~h}$, the membrane was incubated with anti-CLDN3 antibody (1:200, Invitrogen) at $4^{\circ} \mathrm{C}$ overnight. Targeted protein bands were detected by chemiluminescent method with enhanced chemiluminescence detection system (Pierce Biotech Inc., Rockford, IL). $\beta$-actin served as the protein loading control. Bands were analyzed using Quantity One 4.52 software (Bio-Rad).

Intraperitoneal ovarian cancer carcinomatosis model and shRNA treatment. The following study protocols were approved by the Institutional Animal Care and Treatment Committee of the Sichuan University (Chengdu, China 610041). Female athymic BALB/c nude mice, 6-8-week old, were maintained in pathogen-free conditions and given sterile food and water ad libitum. Intraperitoneal carcinomatosis model of ovarian cancer was established referring to a method that had been reported by our laboratory with some modification (23). Briefly, $5 \times 10^{6}$ SKOV3 cells resuspended in $100 \mu 1$ DMEM medium (free of serum and antibiotics) were injected s.c. into the right flank of 3 mice. When the diameter of subcutaneous tumors reached about $10 \mathrm{~mm}$, tumor nodes were 
collected and grinded into small particles $\left(<1 \mathrm{~mm}^{3}\right)$ by sterile stainless screen cloth, and then small particles of tumor tissue were resuspended by serum-free medium to a final volume of $7.5 \mathrm{ml}$ for i.p. inoculation. Fifteen mice were i.p. inoculated with $0.5 \mathrm{ml}$ of above tumor particle suspension, and then randomly allocated into three groups (five per group). Three days after inoculation, mice were i.p. administered with $5 \mu \mathrm{g}$ of PshCLDN3 or PshHK encapsulated in PLGA-NPs once every two days. Mice given with the same volume of NS served as the blank control. Three days after the 12th time of administration, mice were euthanized and tumors were collected and weighed.

Immunohistochemistry staining. Intraperitoneal tumor nodes were collected and divided into two parts. One was fixed in $10 \%$ formalin, and then embedded in paraffin. The other was stored at $-80^{\circ} \mathrm{C}$ for frozen section and protein extraction. Sections (3-5 $\mu \mathrm{m})$ were made from paraffin-embedded intraperitoneal tumors of each group. Immunostaining of CLDN3, Ki67 was carried out by polyclonal antibody of CLDN3 (1:150 Invitrogen) and monoclonal antibody of Ki67 (Maixin Bio, Co., Fujian, China). Cell proliferation index was determined by calculating the ratio of Ki67-positive cells with brown staining nuclei to the total cancer cell number in the same areas (5 random fields of three respective slides per group at $\mathrm{x} 400$ magnification). To determine the angiogenic microvessel density (MVD), frozen sections $(8-10 \mu \mathrm{m})$ of each group were made and stained with antibody of CD31 (1:200, BD Biosciences). Then MVD was determined by examining vascular hot spots at low power (x100) as described previously (24). Vessels with a clearly defined brown-staining lumen or well defined linear vessel shape, were taken into account for blood microvessels. Mean counts of blood microvessels in five fields $(100 \times)$ of three representative slides from each group were recorded.

TUNEL assay for tumor sample. Apoptotic cells in tumor tissue were detected on paraffin sections using terminal deoxy-nucleotidyl transferase-mediated dUTP nick end labeling DeadEnd ${ }^{\mathrm{TM}}$ Fluorometric (TUNEL) system assay kit (Promega, Madison, USA) following the manufacturer's instructions. Sections were observed under a fluorescence microcopy (Olympus BX60, Tokyo, Japan). Cells with pyknotic nucleus of dark green fluorescent staining were defined as TUNEL-positive cells. The apoptotic index was determined by calculated the average percentage of TUNEL-positive cells to the total number of cancer cells in the same field at a magnification of $\mathrm{x} 400$ in three representative sections from each group (Five high-power fields excluding areas of necrosis/section).

Toxicity assessment. To detect possible side effects and toxicity of this treatment, health correlated indexes such as weight loss, diarrhea, anorexia, cachexia, and skin ulcer were monitored during experiment. At the termination of the in vivo study, main organs including heart, liver, spleen, lung and kidney of mice in each group were embedded in paraffin and cut into 3-5- $\mu \mathrm{m}$ paraffin sections. Sections of each organ were detected by HE staining and observed by two experienced pathologists in a blinded manner.
A

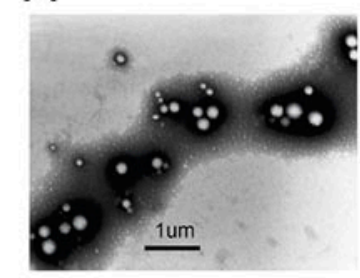

TEM image of PLGA NPS $20,000 \times$

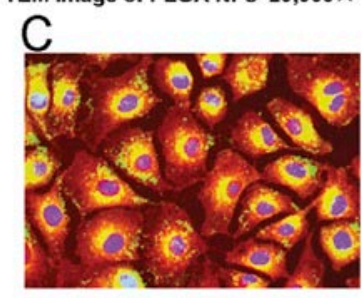

Intracellular uptake of PLGA NPs $400 \times$
B

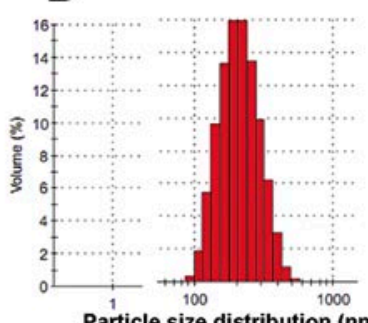

D

Particle size distribution ( $\mathrm{nm}$

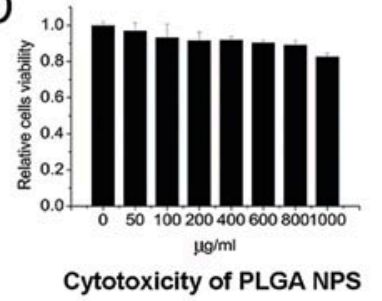

Figure 1. The characteristics of pDNA encapsulating PLGA NPs. (A) Representative TEM (Transmission electron microscope, Hitachi H-600) image of PLGA NPs loaded with pDNA. (B) Particle size and zeta potential of pDNA-PLGA-NPs were determined using Zetasizer Nano ZS90 (Malvern Instrument, UK). Size measurements were performed in triplicates following a $1 / 10(\mathrm{v} / \mathrm{v})$ dilution of the PLGA-NPs suspension in MilliQ water at $25^{\circ} \mathrm{C}$. Zeta potential was measured in the same instrument at $25^{\circ} \mathrm{C}$ using the same protocol. (C) In cell uptake study, cell membrane was prestained with red fluorescence dye for live cell membrane DiI (1,1'-dioctadecyl $-3,3,3^{\prime}, 3^{\prime}$-tetramethyl indocarbocyanine perchlorate), then suspension of 6-coumarin labled pDNA-PLGA-NPs was incubated with cell for $24 \mathrm{~h}$. Cell nuclei were stained with Hoechst 33258 (blue) and washed with PBS twice before observation. Pictures were synthesized by composite Images of three fluorescence channels of red, blue and green. (D) The cytotoxicity of PLGA-NPs was evaluated by methylthiazoletetrazolium (MTT) assay using HEK 293 cells. In brief, cells were seeded at a density of $1 \times 10^{3}$ / cell for HEK-293 in 96-well plates. After $24 \mathrm{~h}$ of culturing, the cells were treated for $48 \mathrm{~h}$ with elevating concentration of empty PLGA NPs. Cell viability were assessed as percent cell viability in terms of media-only treated control cells. Media only treated cells were considered as $100 \%$ cell viability (experiments were performed in triplicate).

Statistical analysis. All numerical experimental values were expressed as means $\pm \mathrm{SD}$. One-way analysis of variance (ANOVA) was employed for multiple comparison among different groups using SPSS software (version 13.0). Differences were considered significant at $\mathrm{P}<0.05$.

\section{Results}

\section{Characteristic of pDNA-PLGA-NPS}

Mean diameter and zeta potential of pDNA-PLGA-NPs. The mean diameter of the pDNA-PLGA-NPs was $218.00 \pm 3.39 \mathrm{~nm}$ with a PDI (polydispersity index) of $0.15 \pm 0.01$ determined by Zetasizer Nano ZS90. Representative TEM (transmission electron microscope) image of pDNA-PLGA-NPs was showed in Fig. 1A. The particle size distribution was showed in Fig. 1B. The zeta potential of the pDNA-PLGA-NPs measured in PBS $(\mathrm{PH}=7.35)$ was $-14.8 \mathrm{mV}$.

Encapsulating efficienciey of PLGA NPs for plasmid. Using synthesis technology above, we achieved a highly and steady encapsulating rate as high as $90 \%$. This indicated that pDNA could be effectively encapsulated into PLGA nanoparticles. 
A

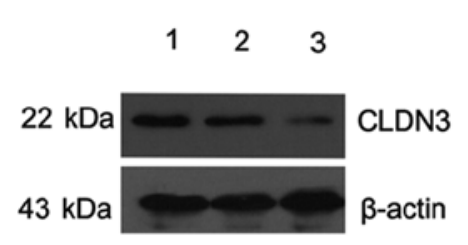

B

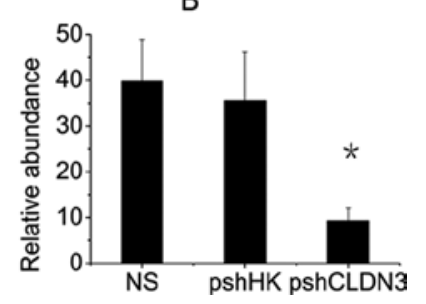

Figure 2. Down-regulation of CLDN3 expression in SKOV3 cells. (A) PshCLDN3 and PshHK were encapsulated into PLGA NPs and then incubated with SKOV3 cells in the presence of serum. Equal volume of NS served as the blank control. Seventy-two hours after transfection, cells were harvested and total protein was extracted for Western blotting to evaluate the change of CLDN3 at protein level. The $\beta$-actin served as the loading internal control. Intact expression of CLDN3 protein was visible in PshHK and NS group. A remarkably reduction of CLDN3 could be observed in cells transfected with PshCLDN3 compared PshHK and NS. (B) The relative CLDN3 expression of each band was normalized to the $\beta$-actin. PshCLDN3 deregulated CLDN3 expression by $72.3 \%$, whereas PshHK had no discernible effect on the expression of CLDN3. Data are represented as mean \pm SD of three independent experiments. The results are presented as mean \pm SD $(\mathrm{n}=3) .{ }^{*} \mathrm{P}<0.05$ vs. pshHK and NS group, respectively.

PLGA NPs internalisation in cells. In order to certify whether PLGA NPs could transport plasmid DNA into cancer cells, we evaluated internalisation of PLGA-NPs in cell culture. Briefly, pDNA-PLGA NPs were labeled with 6-coumarin (green fluorescence), and then incubated with cells that prestained with red fluorescence dye for cellular membrane to outline the profile of cells. After incubating at the presense of serum for $24 \mathrm{~h}$, the pDNA-PLGA-NPs were observed effectively uptaked by cancer cells. The subcellular localization on PLGA-NPs was mainly concentrated in area near cell nucleus (Fig. 1C). This result was coincident with a previous study about PLGA (25). Furthermore, data have suggested that PLGA NPs could rapidly escape the endo-lysosomal pathway and deliver various therapeutic agents into the cytoplasm and nucleus (26). These features of PLGA NPs may be helpful for the gene expression of a plasmid vector.

Toxicity test of PLGA NPs. Cytotoxicity of the PLGA NPs was evaluated by MTT assay using HEK 293 cells. As showed in Fig. 1D, cell viability decreased by the elevating concentration of PLGA NPs. However, the cell viability of HEK 293 cell was still higher than $80 \%$ even at input concentration of PLGA NPs as high as $1 \mathrm{mg} / \mathrm{ml}$. This implied that the PLGA NPs prepared in our study were biocompatible and of low cell cytotoxicity.

PshCLDN3-PLGA-NPs down-regulate the expression of $C L D N 3$ in vitro. To examine whether pshCLDN3 could inhibit CLDN3 expression in vitro, pshCLDN3 and pshHK were encapsulated into PLGA-NPs and then incubated with SKOV3 cells for transfection in 6-well plates at the concentration of $1 \mu \mathrm{g} \mathrm{plasmid/well.} \mathrm{Equal} \mathrm{volume} \mathrm{of} \mathrm{normal}$ sodium served as blank control. After incubation for $72 \mathrm{~h}$, cells were harvested, total protein was extracted for Western blot assay using antibody of CLDN3. As showed in Fig. 2A, Western blotting revealed a remarkably reduction of CLDN3 in cells transfected with PshCLDN3 compared with that in
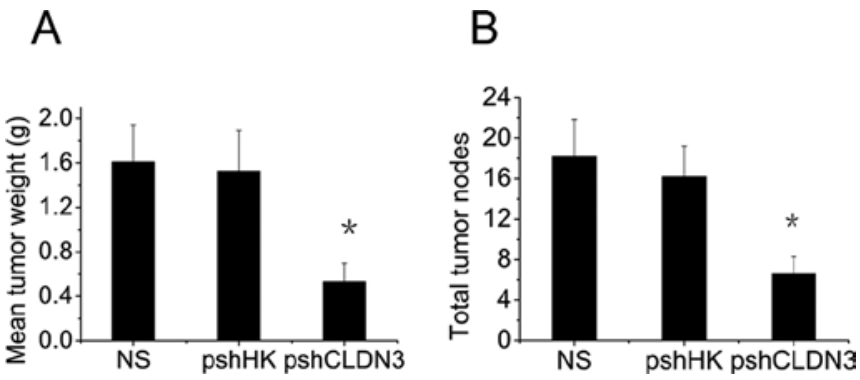

Figure 3. PshCLDN3 inhibited intraperitoneal ovarian tumor growth. For in vivo study, 15 mice were randomly devided into three groups (five per group). Three days after i.p. inoculation) mice were administered i.p. with $10 \mu \mathrm{l}$ plasmids loaded PLGA-NPs solution (contain $5 \mu \mathrm{g}$ of corresponding shRNA coding plasmids, respectively) once every two days. Mice given with the same volume of NS served as the blank control. (A) After 12 times of treatment, mice were sacrificed by cervical dislocation and tumors were harvested and weighted. The mean tumor weight in pshCLDN3-NPs treated mice was $0.532 \pm 0.165 \mathrm{~g}$ vs. $1.61 \pm 0.33 \mathrm{in}$ NS treated mice, and $1.529 \pm 0.367 \mathrm{~g}$ in PshHK-NPs treated mice, respectively. The results are presented as mean $\pm \mathrm{SD}(\mathrm{n}=5) .{ }^{*} \mathrm{P}<0.05$ vs. pshHK and NS treated group. (B) Beside tumor weight, the intraperitoneal tumor node counts of each group were compared. The tumor node counts of pshCLDN3 group exhibited dramatically reduction compared with NS and PshHK-NPs treated group $(\mathrm{P}<0.05)$. Complete tumor regression was observed in one of the five mice administered with pshCLDN3-NPs. ${ }^{*}<<0.05$ vs. pshHK and NS treated group, respectively.

PshHK and blank control. PshCLDN3 deregulated CLDN3 expression by $72.3 \%$ compared with blank control (Fig. 2B), whereas PshHK had no discernible effect on the expression of CLDN3. This result indicated that the shRNA sequence of CLDN3 chosen in our study was specific and effective. PshCLDN3 could efficiently inhibit CLDN3 expression by shRNA meditated RNA interference.

PshCLDN3 inhibited intraperitoneal ovarian tumor growth. In order to evaluate the potential anti-tumor ability of pshCLDN3, we developed an intraperitoneal carcinomatosis model of ovarian cancer in athymic mice and treated them with i.p. injection of pshCLDN3-NPs, PshHK-NPs and NS, respectively. As showed in Fig. 3A, the mean tumor weight (mean $\pm \mathrm{SD}$ ) in pshCLDN3-NPs treated mice was only $0.532 \pm 0.16 \mathrm{~g}$ vs. $1.61 \pm 0.33$ in NS treated mice, and $1.529 \pm 0.36 \mathrm{~g}$ in PshHK-NPs treated mice at the end of treatment. Intraperitoneal administration of pshCLDN3-NPs significantly suppressed tumor growth in comparison with NS and pshHK-NPs $(p<0.05)$, reduced tumor weight by about $67 \%$ compared to the blank control. No significant difference of tumor weight was observed between pshHK-NPs and NS group. Furthermore, we compared the intraperitoneal tumor node counts of each group. As for pshCLDN3 group, the tumor nodes count exhibited dramatically reduction compared with NS and pshHK-NPs groups $(\mathrm{P}<0.05)$ (Fig. 3B). Complete tumor regression was seen in one of the five mice administered with pshCLDN3-NPs.

Down-regulation of CLDN3 expression in vivo. Immunohistochemical staining was employed to compare the expression of CLDN3 in tumor sections from each group. Tumor sections of each group were stained with anti-CLDN3 antibody. As shown in Fig. 4, CLDN3 expression was mainly 


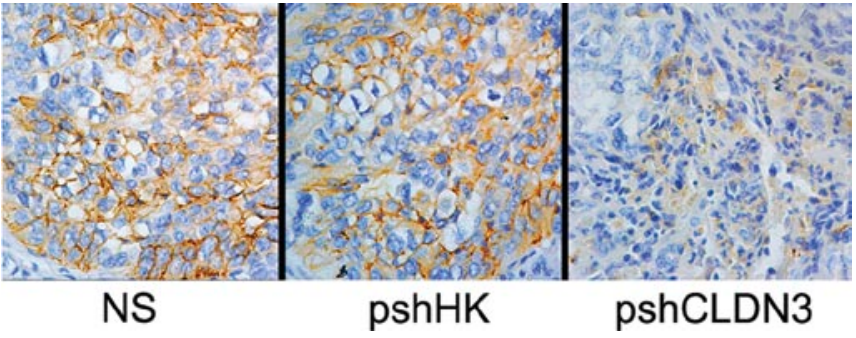

Figure 4. Down-regulation of CLDN3 expression in vivo. Tumors of every group were collected and embedded in paraffin, then 5-8 $\mu \mathrm{m}$ sections were made. Immunochemical staining was used to detect the CLDN3 expression in tumors of each group using antibody CLDN3 (1:100). Representative images of each group were captured by Olympus microscope. Compared with groups of NS and pshHK, the immunostaining intensity of CLDN3 in tumors of pshCLDN3 treated mice were obviously weaker.

located at the cell membrane. Tumor sections from NS and PshHK treated mice demonstrated intensive brown-staining of CLDN3 at cell membrane. On the contrary, the staining of CLDN3 in tumors from pshCLDN3-NPs treated mice was remarkably weaken compared to that of NS and PshHK-NPs. Many pyknotic nucleus and nuclear fragmentation were observed in the background of obviously weaken staining of CLDN3. Western blot assay of total protein extracted from tumor tissues also showed significantly reduced CLDN3 protein consistent with CLDN3 immunostaining of tumor sections (data not shown). These results suggested that the pshCLDN3 encapsulated in PLGA NPs adopted here also suppressed the expression of CLDN3 in vivo, the in vivo antitumor effect was attributed to the down-regulation of CLDN3.

Inhibited proliferation in vivo. To explore potential mechanisms underlying the antitumor effect of CLDN3 silencing in vivo, Ki-67 inmmunostaining was employed to demonstrate change of proliferation in tumor tissues. Cells which were active in proliferation process were characterized by positive staining of Ki-67. Proliferation index was calculated by the ratio of $\mathrm{Ki}-67$ positive cancer cell to the total number of cancer cells at the same fields. Ki-67 staining indicated a dramatically reduced proliferation index of $14.2 \pm 4.9$ in pshCLDN3 treated tumors vs. $34.4 \pm 6.8$ in NS group and $35.2 \pm 8.0$ in PshHK group (Fig. 5A and B). This indicated that knocking down of CLDN3 could lead to decreased proliferation index compared with controls in vivo. It suggested CLDN3 silencing may have antiproliferation effect for ovarian cancer in vivo.

Induction of cell apoptosis in vivo. Abnormal growth of tumor may be regarded as an imbalance between apoptosis and proliferation of cancer cells. In order to explore the possible role of apoptosis induction in vivo, TUNEL assay was utilized to detect apoptotic cells in tumor tissues of each group. TUNEL assay revealed significantly increased TUNEL-positive nuclei (apoptotic cells) in pshCLDN3 treated tumors $(15.8 \pm 2.16)$, than that in pshHK group (3.2 \pm 1.48$)$ and NS group $(2.2 \pm 0.83)(\mathrm{P}<0.05)$ (Fig. $5 \mathrm{C}$ and $\mathrm{D})$. This indicated
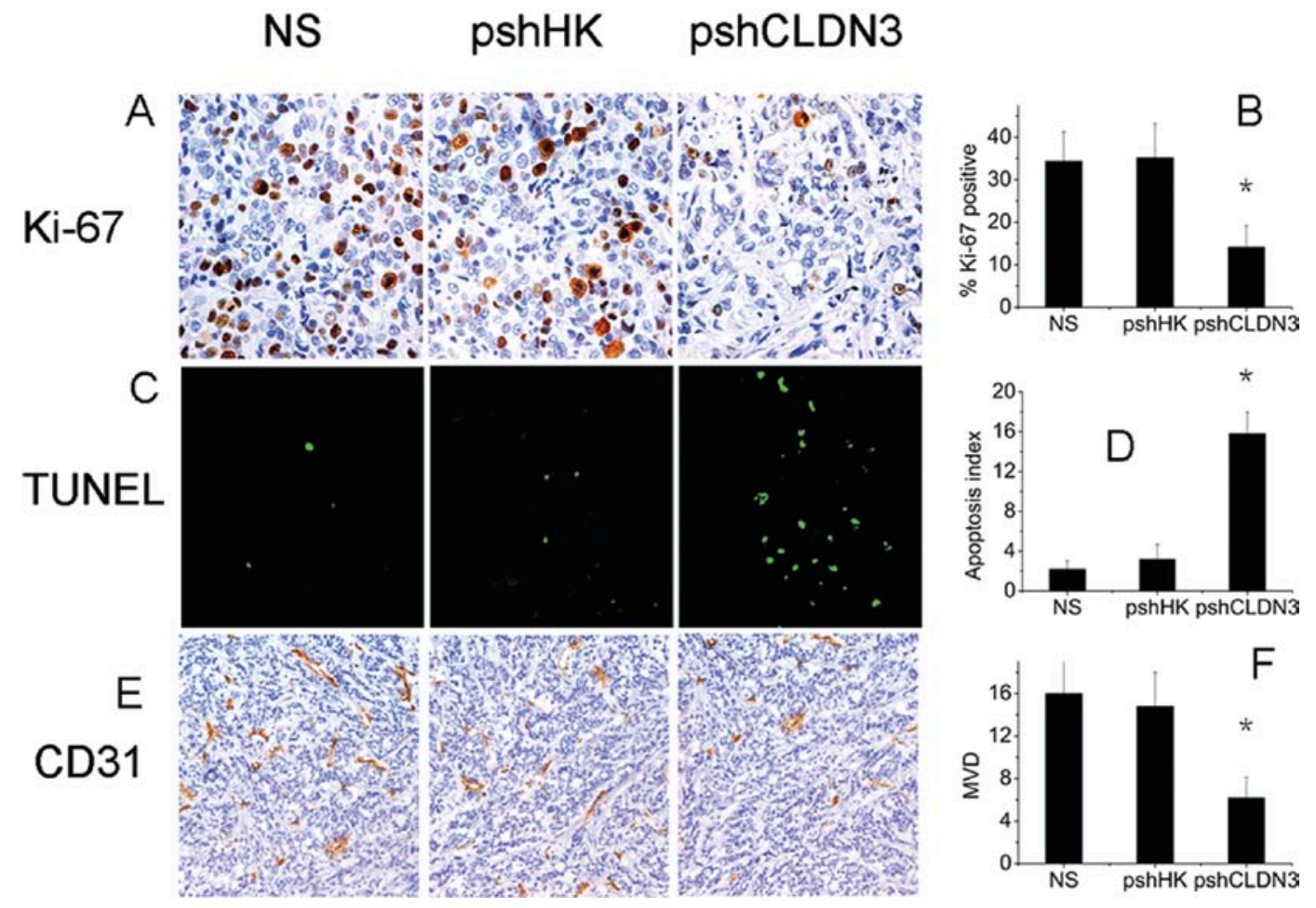

Figure 5. Immunostaining of CD31, Ki-67 and TUNEL assay. (A) Tumor sections immunostained with antibody against Ki-67 (magnification, x400). (B) Quantification of Ki-67 postive cells. (C) Tumor sections stained for TUNEL (magnification, x400). (D) Quantification of TUNEL staining (apoptotic cell numbers). Apoptotic index was obtained by counting the number of the apoptotic cells divided by the total number of cancer cells in the same field. (E) Tumor sections immunostained with antibody against CD31 (magnification, x100). The number of vessels per field (magnification, x100) was calculated. (F) Quantification of vessels. Data are presented as mean \pm SD. ${ }^{*} \mathrm{P}<0.05$ vs. pshHK and NS treated group, respectively. 
that PshCLDN3 could also effectively induce cancer cell apoptosis as one of its antitumor mechanisms.

Inhibited angiogenesis in vivo. One of the most necessary factors for tumor growth is the ability of tumor to stimulate nascent angiogenesis $(27,28)$. Antiangiogenesis is a general mechanism for cancer therapy $(29,30)$. Here, frozen sections were stained by antibody of CD31 to investigate the antiangiogenic effect of pshCLDN3. MVD was evaluated in the tumor sections of each group. CD31 staining demonstrated a significant reduction of MVD in the sections of pshCLDN3 treated group $(6.2 \pm 1.92)$ compared to NS group $(16 \pm 3.16)$ and PshHK treated group $(14.8 \pm 3.19)(\mathrm{P}<0.05)$ (Fig. 5E and F). Numerous newborn blood capillaries were observed in NS group and PshHK group. No significant difference of MVD was found between NS group and PshHK group. Based on this, we may conclude that the antitumor ability of pshCLDN3 is partially due to the inhibition of tumor neovascularization in ovarian cancer.

Toxicity evaluation. No gross abnormalities were observed in mice of each group during treatment process. Furthermore, at the termination of the experiment, $\mathrm{H} \& \mathrm{E}$ histological staining of the heart, liver, spleen, lung and kidney, observed by two pathologists, did not exhibit any significant toxicity associate pathologic alteration in pshCLDN3 treated group compared with the blank control group.

\section{Discussion}

Despite much progress in tumor management during recent years, ovarian cancer remains the most lethal disease of gynecological cancers. Various new chemotherapeutics have been developed for the treatment of ovarian cancer, of which an unavoidable shortcoming is the lack of targeting. Chemotherapeutics generally act on multiple organs and tissues beyond tumors, inducing various unwanted serious side-effects during treatment. Therefore, new anti-tumor strategies with better efficiency and high specificity are expected.

Gene therapy is a novel emerging therapeutic strategy. It exerts remarkable effect on gene related diseases by up-regulating or down-regulating the expression of specific genes through diverse molecular mechanisms. RNAi is a newly discovered gene down-regulating mechanism which could efficiently and specifically suppress target gene at transcriptional level, making it a promising approach for gene therapy. RNAi could be achieved both by synthesized siRNA or shRNA expressed by plasmid. Plasmid vectormediated shRNA is preferred for its simple preparation, relative stability and sustainable expression while compared to siRNA. Therefore, it has been utilized in many geneinvovled fields especially in anti-cancer studies. Specific gene suppression and following tumor growth inhibition elicited by plasmid vector-mediated shRNA have been observed in various animal tumor models (31-33). But homologous reports on ovarian cancer are rare. Therefore, in the present study, we adopted a plasmid vector expressing shRNA targeted CLDN3 to explore a superior therapeutic strategy for ovarian cancer.
CLDN3 has been proved to be one of the most highly and generally up-regulated genes in human ovarian cancer but not in non-malignant disease of the ovary $(15,34)$. Its overexpression has been identified relating to migration, invasion and proliferation of cancer cells $(16,19,35)$. Interestingly, CLDN3 is identified as one of the only two claudin family members capable of mediating CPE binding and cytolysis (36). Currently, most of the studies on CLDN3 are focusing on CPE meditated cytolysis in tumor cells. However, CLDN3 is also postive in several other normal organs and tissues (37). Systemic administration of CPE or siRNA would induce unwanted cytolysis of normal cells expressing CLDN3, interfere with the expression of CLDN3 beyond tumors, thus, hamper normal organs function, impair the specificity of gene therapy and cause numerous unpredictable side effects in return. Therefore, in this study, we took the i.p. route for pshCLDN3-NPs administration to eliminate possible side-effects of systemic administration. Toxicity assessment showed no obvious toxicity in pshCLDN3 treated cells. Specific down-regulation of CLDN3 by the effect of RNAi was confirmed by both Western blot analysis and immunohistochemistry of CLDN3.

Although many gene therapies have demonstrated exciting results in experimental models by using cationic liposomes or virus vectors as gene carriers, these gene carriers suffer from the intrinsic defect of significant toxcity, immunogenicity, potential carcinogenicity and dramatic reduced efficiency in the presence of serum (38), which hinder their applications in clinic. Nanoparticles based on PLGA have been suggested as an ideal carrier for gene therapy (39). Besides the biodegradability and biocompatibility (40), nanoparticles are relatively easy to manipulate enabling us to prepare NPs with desired size and release profile from days to months by regulating the ratio of lactic acid to glycolic acid in copolymer and other variables to meet various needs. Here, we adopted PLGA NPs for gene delivery and prepared PLGA NPs with a mean particle size about $200 \mathrm{~nm}$. It has been shown that nanoparticles with sub-micron size had relatively higher intracellular uptake compared to microparticles (41). In cell uptake test, we observed PLGA NPs were efficiently taked up by cancer cells, and aggregated around the cellular nucleus. These features may provide convenience for the gene expression by the easy access to necessary factors for DNA synthesis around the cellular nucleus. Studies have indicated that blood vessels in different tumors often have a discontinuous endothelium, with pores between the cells about $100 \mathrm{~nm}$ apart (42). Given this, PLGA NPs with suitable particle size may have the potential to extravasate through 'pores' or 'defects' of the capillary endothelium and accumulate in tumor site when administered through intravenous injection implying PLGA NPs could be utilized as a powerful vehicle with passive targeting for treatment of cancer metastasis.

No obvious cytotoxicity of PLGA NPs was observed in MTT assay, and no pathological change was found in HE staining of main organs from PLGA-NPs treated mice indicating that this regimen was apparently well tolerated by the mice.

In this study, pshCLDN3 NPs showed significant antitumor effect in an intraperitoneal ovarian cancer model by i.p administration. Immunostaining demonstrated significant 
weaker CLDN3 expression of tumors from pshCLDN3 NPs treated mice, while pshHK showed equivalent strong positive CLDN3. This suggested the treatment efficiency was due to the down-regulating of CLDN3 instead of off-target effect RNAi. Subsequently, the down-stream mechanisms were systematically analyzed by immnostaining of CD31, Ki-67 and TUNEL assay, respectively. pshCLDN3 showed comprehensive antitumor mechanism including angiogenesis restrain, proliferation inhibition and apoptosis induction. Besides tumor weight, tumor nodes in pshCLDN3 treated group was significantly reduced compared to that of NS and pshHK groups. We infered that this was partially due to the inhibition of invasion potential by knocking down CLDN3, which was consistent with the results of Agarwal et al (16). This may provide an explaination for an interesting phenomenon in vivo: when compared with the isolated and dissociative tumor nodes in pshCLDN3 NPs treated group, the tumors in NS or pshHK-NPs group were conglobate and adhesive with abdominal organs.

Furthermore, in ovarian cancer, which is mainly limited in the abdominal cavity, systemic administration usually could not attain desired drug concentration at the tumor site. Compared with i.v. injection, intraperitoneal administration of gene loaded PLGA NPs avoids the systemic toxicity and first pass effect (43), refrains from clearance through bonding with plasma protein or phagocytized by lymphocyte that was inevitable in cationic lipid-DNA complexes, and maintain high focal concentration of drug in the area where it was topically applied. Furthermore, PLGA NPs released the encapsulated drugs slowly, which led to prolonged exposure of tumor cells and enhanced antitumor effect. Combined with the steerable releasing profile of PLGA NPs, we can prepare gene laoded PLGA NPs with various releasing profile that is suitable for different diseases and patients, to realize the purpose of individual treatment. As far as we know, this is the first study using intraperitoneal administration of gene loaded PLGA NPs for ovarian cancer.

In summary, this study showed that pshCLDN3 could efficiently inhibit CLDN3 expression in vitro, and significant tumor growth inhibition was achieved after down-regulating CLDN3 by i.p. administration of pshCLDN3 in PLGA NPs. These data indicated that i.p. administration of pshCLDN3 meditated by PLGA NPs might provide a promising approach for the treatment of ovarian cancer.

\section{Acknowledgements}

This study was financially supported by National 973 Program of China grants (2011CB910703, 2010CB529905).

\section{References}

1. Jemal A, Siegel R, Xu J and Ward E: Cancer statistics, 2010. CA Cancer J Clin 60: 277-300, 2010.

2. DiSaia PJ and Creasman WT: Epithelial ovarian cancer. In: Clinical Gynecologic Oncology. 5th edition. DiSaia PJ and Creasman WT (eds). Mosby, St. Louis, 1997.

3. Jemal A, Siegel R, Ward E, Hao Y, Xu J and Thun MJ: Cancer statistics, 2009. CA Cancer J Clin 59: 225-249, 2009.

4. Clark PR and Hersh EM: Cationic lipid-mediated gene transfer: current concepts. Curr Opin Mol Ther 1: 158-176, 1999.
5. Cristiano RJ: Viral and non-viral vectors for cancer gene therapy. Anticancer Res 18: 3241-3245, 1998.

6. Merdan T, Kopecek J and Kissel T: Prospects for cationic polymers in gene and oligonucleotide therapy against cancer. Adv Drug Deliv Rev 54: 715-758, 2002.

7. Jabbarzadeh E, Starnes T, Khan YM, et al: Induction of angiogenesis in tissue-engineered scaffolds designed for bone repair: a combined gene therapy-cell transplantation approach. Proc Natl Acad Sci USA 105: 11099-11104, 2008.

8. Hao T, McKeever U and Hedley ML: Biological potency of microsphere encapsulated plasmid DNA. J Control Release 69: 249-259, 2000.

9. Panyam J, Zhou WZ, Prabha S, Sahoo SK and Labhasetwar V: Rapid endo-lysosomal escape of poly(DL-lactide-co-glycolide) nanoparticles: implications for drug and gene delivery. FASEB J 16: 1217-1226, 2002.

10. Li Z and Huang L: Sustained delivery and expression of plasmid DNA based on biodegradable polyester, poly(D,L-lactide-co-4hydroxy-L-proline). J Control Release 98: 437-446, 2004.

11. Song X,Zhao Y,Wu W, et al: PLGA nanoparticles simultaneously loaded with vincristine sulfate and verapamil hydrochloride: systematic study of particle size and drug entrapment efficiency. Int J Pharm 350: 320-329, 2008.

12. Song XR, Cai Z, Zheng Y, et al: Reversion of multidrug resistance by co-encapsulation of vincristine and verapamil in PLGA nanoparticles. Eur J Pharm Sci 37: 300-305, 2009.

13. Morita K, Furuse M, Fujimoto K and Tsukita S: Claudin multigene family encoding four-transmembrane domain protein components of tight junction strands. Proc Natl Acad Sci USA 96: 511-516, 1999.

14. Hough CD, Sherman-Baust CA, Pizer ES, et al: Large-scale serial analysis of gene expression reveals genes differentially expressed in ovarian cancer. Cancer Res 60: 6281-6287, 2000.

15. Rangel LB, Agarwal R, D'Souza T, et al: Tight junction proteins claudin-3 and claudin- 4 are frequently overexpressed in ovarian cancer but not in ovarian cystadenomas. Clin Cancer Res 9: 2567-2575, 2003.

16. Agarwal R, D'Souza T and Morin PJ: Claudin-3 and claudin-4 expression in ovarian epithelial cells enhances invasion and is associated with increased matrix metalloproteinase-2 activity. Cancer Res 65: 7378-7385, 2005.

17. Romani C, Comper F, Bandiera E, et al: Development and characterization of a human single-chain antibody fragment against claudin-3: a novel therapeutic target in ovarian and uterine carcinomas. Am J Obstet Gynecol 201: 70.e71-79, 2009.

18. Santin AD, Cane S, Bellone S, et al: Treatment of chemotherapyresistant human ovarian cancer xenografts in C.B-17/SCID mice by intraperitoneal administration of Clostridium perfringens enterotoxin. Cancer Res 65: 4334-4342, 2005.

19. Huang YH, Bao Y, Peng W, et al: Claudin-3 gene silencing with siRNA suppresses ovarian tumor growth and metastasis. Proc Natl Acad Sci USA 106: 3426-3430, 2009.

20. Freytag T, Dashevsky A, Tillman L, Hardee GE and Bodmeier R: Improvement of the encapsulation efficiency of oligonucleotidecontaining biodegradable microspheres. J Control Release 69: 197-207, 2000.

21. Cui FY, Song XR, Li ZY, et al: The pigment epithelial-derived factor gene loaded in PLGA nanoparticles for therapy of colon carcinoma. Oncol Rep 24: 661-668, 2010.

22. Song X, Zhao Y, Hou S, et al: Dual agents loaded PLGA nanoparticles: systematic study of particle size and drug entrapment efficiency. Eur J Pharm Biopharm 69: 445-453, 2008.

23. Lin XJ, Chen XC, Wang L, et al: Dynamic progression of an intraperitoneal xenograft model of human ovarian cancer and its potential for preclinical trials. J Exp Clin Cancer Res 26: 467-474, 2007.

24. Mukherjee P, Abate LE and Seyfried TN: Antiangiogenic and proapoptotic effects of dietary restriction on experimental mouse and human brain tumors. Clin Cancer Res 10: 5622-5629, 2004.

25. Cohen H, Levy RJ, Gao J, et al: Sustained delivery and expression of DNA encapsulated in polymeric nanoparticles. Gene Ther 7: 1896-1905, 2000.

26. Ando S, Putnam D, Pack DW and Langer R: PLGA microspheres containing plasmid DNA: preservation of supercoiled DNA via cryopreparation and carbohydrate stabilization. J Pharm Sci 88: 126-130, 1999.

27. Folkman J: Tumor angiogenesis: therapeutic implications. N Engl J Med 285: 1182-1186, 1971. 
28. Li CY, Shan S, Huang Q, et al: Initial stages of tumor cellinduced angiogenesis: evaluation via skin window chambers in rodent models. J Natl Cancer Inst 92: 143-147, 2000.

29. Folkman J: Angiogenesis: an organizing principle for drug discovery? Nat Rev Drug Discov 6: 273-286, 2007.

30. Isner JM and Asahara T: Angiogenesis and vasculogenesis as therapeutic strategies for postnatal neovascularization. J Clin Invest 103: 1231-1236, 1999.

31. Paddison PJ, Caudy AA, Bernstein E, Hannon GJ and Conklin DS: Short hairpin RNAs (shRNAs) induce sequencespecific silencing in mammalian cells. Genes Dev 16: 948-958, 2002.

32. Wannenes F, Ciafre SA, Niola F, Frajese G and Farace MG: Vector-based RNA interference against vascular endothelia growth factor-A significantly limits vascularization and growth of prostate cancer in vivo. Cancer Gene Ther 12: 926-934, 2005.

33. Zhang Y, Zhang YF, Bryant J, Charles A, Boado RJ and Pardridge WM: Intravenous RNA interference gene therapy targeting the human epidermal growth factor receptor prolongs survival in intracranial brain cancer. Clin Cancer Res 10: 3667-3677, 2004

34. He Q, Liu J, Sun X and Zhang ZR: Preparation and characteristics of DNA-nanoparticles targeting to hepatocarcinoma cells. World J Gastroenterol 10: 660-663, 2004.

35. Morin PJ: Claudin proteins in human cancer: promising new targets for diagnosis and therapy. Cancer Res 65: 9603-9606, 2005 .
36. Katahira J, Sugiyama H, Inoue N, Horiguchi Y, Matsuda M and Sugimoto N: Clostridium perfringens enterotoxin utilizes two structurally related membrane proteins as functional receptors in vivo. J Biol Chem 272: 26652-26658, 1997.

37. Kiuchi-Saishin Y, Gotoh S, Furuse M, Takasuga A, Tano Y and Tsukita S: Differential expression patterns of claudins, tight junction membrane proteins, in mouse nephron segments. J Am Soc Nephrol 13: 875-886, 2002.

38. Zelphati O, Uyechi LS, Barron LG and Szoka FC Jr: Effect of serum components on the physico-chemical properties of cationic lipid/oligonucleotide complexes and on their interactions with cells. Biochim Biophys Acta 1390: 119-133, 1998.

39. Diez S and Tros de Ilarduya C: Versatility of biodegradable poly(D,L-lactic-co-glycolic acid) microspheres for plasmid DNA delivery. Eur J Pharm Biopharm 63: 188-197, 2006.

40. Pan H, Jiang H and Chen W: The biodegradability of electrospun Dextran/PLGA scaffold in a fibroblast/macrophage co-culture. Biomaterials 29: 1583-1592, 2008.

41. Panyam $\mathbf{J}$ and Labhasetwar V: Biodegradable nanoparticles for drug and gene delivery to cells and tissue. Adv Drug Deliv Rev 55: 329-347, 2003.

42. Jain RK: Transport of molecules in the tumor interstitium: a review. Cancer Res 47: 3039-3051, 1987.

43. Howell SB: Selection of drugs for intraperitoneal chemotherapy for ovarian cancer. In: Intraperitoneal Therapy for Ovarian Cancer. Alberts DS, Clouser MC and Hess LM (eds). SpringerVerlag, Heidelberg, pp77-88, 2010. 